\title{
REACCIÓN DE VARIEDADES DE FRIJOL A LA INOCULACIÓN ARTIFICIAL DE Aphelenchoides besseyi, AGENTE CAUSAL DE LA FALSA MANCHA ANGULAR ${ }^{1}$
}

\author{
Walter Barrantes-Santamaría ${ }^{2}$, Carlos Manuel Araya-Fernández ${ }^{3}$
}

\begin{abstract}
RESUMEN
Reacción de variedades de frijol a la inoculación artificial de Aphelenchoides besseyi, agente causal de la falsa mancha angular. Especímenes adultos y juveniles de A. besseyi provenientes de cultivo in vitro se inocularon sobre el follaje de plantas de frijol de las variedades Bribri, Huetar, Telire y Chirripó Rojo. Las edades de las plantas inoculadas en el invernadero fueron de diez, 20 y 30 días después de siembra (dds). Los bioensayos se realizaron en los invernaderos de la Escuela de Ciencias Agrarias de la Universidad Nacional, Heredia-Costa Rica en el 2006. El objetivo de esta investigación fue evaluar la reacción de variedades de frijol al nematodo A. besseyi. Las variedades estudiadas mostraron similar nivel de resistencia al ataque del nematodo, de acuerdo a la escala de severidad utilizada; sin embargo, las variedades Huetar y Bribri mostraron menor severidad de infección y población de nematodos que las otras dos variedades. Los primeros síntomas de la enfermedad se desarrollaron en las hojas primarias. El período de incubación fue más corto a medida que las inoculaciones se realizaron en plantas de mayor edad. Igualmente, las hojas maduras fueron más susceptibles que las jóvenes a la infección del nematodo. El desarrollo de la enfermedad difirió entre hojas primarias y trifoliadas y dependió de la disponibilidad de tejido foliar, la madurez del follaje y la producción de inóculo secundario.
\end{abstract}

Palabras claves: Phaseolus vulgaris, frijol común, nematodos, incidencia, severidad.

\begin{abstract}
Reaction of bean varieties to artificial inoculation of Aphelenchoides besseyi, causal agent of the false angular leaf spot. The objective of this work was to evaluate the reaction of common bean varieties to the nematode Aphelenchoides besseyi. Bioassays were done in greenhouses, National University, Heredia-Costa Rica in the 2006. Adult and juvenile instars of the nematode were inoculated on four bean varieties (Bribri, Huetar, Telire and Chirripó rojo), 10, 20 and 30 days after seeding. Plants were kept under favourable relative humidity, light and temperature conditions, in a greenhouse. All bean varieties showed a similar resistant level to nematode infection; however, Huetar and Bribri varieties presented lower infection rate and nematode population than the other two bean varieties. The first symptoms were observed on the primary leaves. The incubation period decreased as the plants aged. Likewise, mature leaves were more susceptible to nematode infection than young ones. The disease progress differed between primary and trifoliated leaves according to host tissue availability, maturity of leaves, and secondary inoculum production.
\end{abstract}

Key words: Phaseolus vulgaris, common bean, nematode, incidence, severity.

\section{INTRODUCCIÓN}

Lafalsa mancha angular(FMA) es una enfermedad del frijol, que en los últimos años ha experimentado un considerable aumento en la incidencia y severidad en las principales zonas productoras del país (Araya y Hernández 2003, Barrantes y Araya 2004). A pesar que en los Estados Unidos se tiene a Aphelenchoides

\footnotetext{
Recibido: 20 de setiembre, 2007. Aceptado: 24 de enero, 2008.

2 Estación Experimental Fabio Baudrit Moreno, Universidad de Costa Rica. Alajuela, Costa Rica. Correo electrónico: wbarran@cariari.ucr.ac.cr

3 Universidad Nacional (UNA). Heredia, Costa Rica. Correo electrónico: caraya@una.ac.cr
} 
ritzemabosi Schwartz 1911 como el agente causal (Franc et al. 1996) estudios realizados en Costa Rica demuestran que la especie A. besseyi Christie 1942 es la responsable de la enfermedad (Salas y Vargas 1984). Los síntomas que causa en frijol son similares a los encontrados en otros hospedantes; éstos inician con pequeñas manchas cloróticas en el follaje, que luego aumentan de tamaño y se tornan pardo oscuro, rodeadas de un tenue halo clorótico. Son de forma angular debido a que las venas limitan el avance del nematodo. El tamaño de las lesiones varía de 0,2 a $1,5 \mathrm{~cm}$ de diámetro, con coalescencia. En estados avanzados de parasitismo puede ocurrir necrosis en toda la hoja, acelerando la senescencia foliar (Salas y Vargas 1984, Franc et al. 1996).

Dado que los esfuerzos por conocer sobre la epidemiología de la FMA del frijol son recientes, no se cuenta con estudios sobre la reacción de las variedades comerciales de frijol al nematodo. Evaluaciones de campo han mostrado que la mayoría de las variedades comerciales son susceptibles al nematodo (Araya y Hernández 2007). Además, no se tiene información confiable sobre el efecto que tiene el momento de la infección (etapa de desarrollo del cultivo) en la incidencia y severidad de la enfermedad, así como sobre el rendimiento.

El objetivo de esta investigación fue conocer la reacción de variedades comerciales de frijol al nematodo Aphelenchoides besseyi bajo condiciones de ambiente controlado, con miras a establecer efectivamente el nivel de resistencia en el germoplasma comercial de Costa Rica.

\section{MATERIALES Y MÉTODOS}

El ensayo se realizó en los invernaderos de la Escuela de Ciencias Agrarias de la Universidad Nacional, ubicados en el Campus Omar Dengo, Heredia, Costa Rica, en el año 2006. Las variedades de frijol evaluadas fueron Bribri, Telire, Chirripó Rojo y Huetar (Araya y Hernández 2007).

Para el incremento del inóculo, los nematodos fueron extraídos de follaje de frijol con síntomas de FMA y multiplicados in vitro en medio de cultivo papa dextrosa agar (PDA) + Fusarium oxysporum (Barrantes et al. 2005), luego se extrajeron del medio a través de la técnica de Baerman (CIAT 1982a). Una suspensión de 20 nematodos por $\mathrm{ml}$ fue asperjada uniformemente sobre el follaje. Para garantizar la distribución homogénea de los nematodos, la suspensión se mantuvo en agitación durante la inoculación. Se inocularon aproximadamente 120 nematodos (juveniles y adultos) por planta.

Los nematodos se inocularon sobre plántulas de frijol en tres estados fenológicos V2 (hojas primarias totalmente desplegadas); V4 (tercer hoja trifoliadas totalmente formada) y R5 (etapa de prefloración) a los diez, 20 y 30 días de edad, respectivamente (CIAT 1982b). Las plantas inoculadas se mantuvieron en un invernadero de techo transparente y paredes de malla, en donde permanecieron entre 45 y 65 días, dependiendo de la edad en que fueron inoculadas. Se favorecieron condiciones de alta humedad relativa, por medio de riego por aspersión (descargas de 15 seg. cada $10 \mathrm{~min}$ ), que mantuvo el follaje siempre mojado y la temperatura alrededor de $24^{\circ} \mathrm{C}$.

Los tratamientos consistieron de combinaciones de las variedades de frijol (Bribri, Huetar, Telire y Chirripó Rojo) (Araya y Hernández 2007) por estado de desarollo (V2, V4 y R5) dispuestos en bloques al azar con un arreglo factorial $4 * 3$, y cuatro repeticiones. La unidad experimental estuvo constituida por tres plantas, sembradas en macetas con una capacidad volumétrica de $11 \mathrm{t}$, con suelo estéril. Las evaluaciones se iniciaron a partir de la aparición de los síntomas de la enfermedad. Se determinó la duración del período de incubación, el cual constituyó el lapso de tiempo transcurrido desde la inoculación de los nematodos hasta la aparición de las primeras lesiones, se evaluó la incidencia de la FMA; para evaluar la severidad se utilizó una escala de 0 a 9, donde 0 es ausencia de la enfermedad y 9 más del $25 \%$ de la hoja afectada. Finalmente, se contabilizó el número de especímenes A. besseyi en $10 \mathrm{~g}$ de tejido foliar. Para la extracción de los nematodos se utilizó la técnica de Baermann (CIAT 1982a).

A los datos obtenidos se les realizó un ANDEVA y la comparación de medias (DMS 0,05) cuando hubo diferencias significativas. 


\section{RESULTADOS Y DISCUSIÓN}

\section{Período de incubación}

De acuerdo con los datos del periodo de incubación registrados en el ensayo, el inicio del ataque de $A$. besseyi a las plantas de frijol, varió según el tipo de hoja y el estado de desarollo en que la planta fue inoculada (Cuadro 1). El análisis de medias a los datos de período de incubación determinó diferencias estadísticas entre las edades de inoculación y entre tipos de hojas, no así entre las variedades $(\mathrm{p}<0,05)$ (Cuadro 1$)$.

Cuadro 1. Periodos de incubación promedio de la falsa mancha angular, Plantas de variedades comerciales de frijol inoculadas en tres estados de desarrollo*. UNA, Heredia, Costa Rica, 2006.

\begin{tabular}{cc}
\hline Variedades & Periodo de incubación \\
\hline Bribri & $11,8 \mathrm{a}$ \\
Huetar & $13,0 \mathrm{a}$ \\
Telire & $9,2 \mathrm{a}$ \\
Chiripo Rojo & $10,4 \mathrm{a}$ \\
\hline Estado de desarrollo & \\
\hline V2 & $9,5 \mathrm{a}$ \\
V4 & $12,7 \mathrm{c}$ \\
R5 & $10,2 \mathrm{~b}$ \\
\hline Tipo de hoja & \\
\hline Primaria & $8,5 \mathrm{a}$ \\
Trifoliada & $15,0 \mathrm{~b}$ \\
\hline
\end{tabular}

* Columnas con diferente letra difieren estadísticamente, según prueba de LSD $(\mathrm{p}<0,05)$

El nematodo inició el parasitismo de la planta en el tejido primario a partir del cual colonizó las hojas trifoliadas; además, tuvo afinidad por tejido maduro. La poca disponibilidad de follaje trifoliado, en algunas de las etapas de desarrollo iniciales que se evaluaron, influyó en la duración del periodo de incubación en este tejido. Asimismo, el desarrollo temprano de síntomas en hojas trifoliadas en la etapa de prefloración (R5) podría estar relacionado, como lo menciona Arauz (1998), con los cambios en la dirección y la redistribución de los recursos energéticos (fotosintatos) hacia los sumideros de mayor actividad. En consecuencia, los estados reproductivos del cultivo pueden aumentar la predisposición de la planta, o de una parte de ella, a la infección por organismos fitopatógenos.

Varios autores (Salas y Vargas 1984, Franc et al. 1996, Hoshino y Togashi 2000) han manifestado que los períodos de incubación para las especies de Aphelenchoides fitoparásitas se ubican entre seis y 15 días. Para la especie A. besseyi en particular, se mencionan periodos de incubación de seis y diez días, en los cultivos de frijol y arroz, respectivamente. El periodo de incubación de ocho días obtenido en este ensayo en hojas primarias, es ligeramente menor a los mencionados por Lin et al. (1992) para otros cultivos parasitados por A. besseyi. Los períodos de incubación relativamente más cortos obtenidos en este ensayo, son consecuencia de las condiciones ideales para el establecimiento y reproducción del nematodo (Cuadro 1). La alta humedad relativa, la temperatura $20-25^{\circ} \mathrm{C}$, y el abundante tejido susceptible, fueron factores que favorecieron la actividad del nematodo.

\section{Síntomas de la enfermedad}

Las lesiones iniciales provocadas por el nematodo presentaron formas de figuras geométricas, que obedecen al arreglo espacial de las nervaduras de las hojas. Lo anterior concuerda con los síntomas descritos por Salas y Vargas (1984) y Franc et al. (1996), en plantas de frijol parasitadas por las especies $A$. besseyi y A. ritzemabosi, respectivamente. Estos autores indican que las nervaduras limitan el avance del nematodo. Las lesiones presentaron un ligero halo clorótico, que según Wallace (1960), es el sitio de avance de la enfermedad, por consiguiente, en esta región se localiza el mayor número de nematodos. Las regiones del tejido próximas a las lesiones, que aún son de color verde, son abundantes en huevos del nematodo; en las regiones necrosadas de las lesiones no se logran observar nematodos (Todd y Atkins 1958).

Los nematodos se ubicaron inicialmente en regiones de la hoja con mayor acumulación de humedad, como son la base del foliolo, ambos lados de la nervadura central, y los bordes de la hoja. Según Wallace (1960), Klingler (1970), Christie (1974) y Agrios (1985), los nematodos que atacan órganos aéreos, necesitan de una 
película de agua sobre los tejidos (hojas y tallos) que utilizan como vehículo para poder desplazarse y lograr ascender hasta órganos superiores de la planta.

En todas las variedades estudiadas, los primeros síntomas siempre aparecieron en las hojas primarias, independientemente del estado de desarrollo en que fueron inoculadas, esto a pesar que ya existían hojas trifoliadas en las etapas V4 y R5. Era de esperarse que gran parte de los nematodos inoculados se depositaran sobre las hojas trifoliadas, dada su ubicación en el dosel superior. En relación con este comportamiento, Wallace (1960) estudió la presencia de A. ritzemabosi en dos variedades de crisantemo y determinó que las diferencias en textura y tamaño de la hojas, así como el número de ramas y longitud de los entrenudos determinaron la presencia de nematodos en una variedad con respecto a la otra. En nuestro caso, la posición horizontal de las hojas primarias permitió, en primera instancia, la captura de especímenes y en segunda instancia, favoreció la permanencia de humedad, que creó un ambiente adecuado para el establecimiento del nematodo, circunstancias que probablemente no se presentaron en hojas trifoliadas por su orientación ligeramente vertical.

\section{Incidencia de FMA en condiciones de invernadero}

El análisis estadístico no mostró diferencias significativas entre variedades en cuanto a la variable incidencia. La incidencia de la enfermedad aumentó en forma proporcional a la edad de la planta en que fue inoculada. Es decir, el tejido juvenil es menos susceptible al nematodo con respecto al tejido maduro. Por otra parte, la mayor incidencia de la enfermedad en hojas primarias confirma lo expuesto anteriormente, donde se afirmó que el nematodo prefiere tejido maduro para iniciar el parasitismo (Figura 1).

Las plántulas inoculadas a los diez días de edad (V2), desarrollaron síntomas solamente en las hojas primarias, debido a que al momento de la inoculación fue el tipo de hojas presentes; las hojas trifoliadas que desarrolló posteriormente, no presentaron síntomas. Pero, considerando que A. besseyi tiene la habilidad de ascender hacia órganos superiores de la planta, ayudado por la película de agua que se acumula en los tejidos en condiciones de alta humedad (Wallace
1960, Klingler 1970, Christie 1974 y Agrios 1985), estas hojas debieron haber presentado síntomas. Es muy probable que el inóculo secundario, en este caso en particular no se produjera en la cantidad suficiente dada la inmadurez del tejido disponible (plántulas de diez días), lo que reafirmaría una vez más que el nematodo prefiere tejido maduro para iniciar el parasitismo y realizar su reproducción.

De acuerdo con los datos obtenidos, las variedades de frijol estudiadas pueden ser afectadas por $A$. besseyi dependiendo del momento en que el patógeno infecta las plantas. Así, la enfermedad podría ser leve cuando el nematodo entra en contacto con la planta en las etapas iniciales de su desarrollo, debido a la inmadurez del tejido, y el daño se limitará a las hojas primarias. Por el contrario, cuando los nematodos logran colonizar las plantas en etapas de mayor madurez, la reproducción y posterior colonización de estratos superiores del dosel se verá favorecida.

\section{Severidad de FMA en condiciones de invernadero}

Los índices de severidad obtenidos en este ensayo no presentaron diferencias estadísticas altamente significativas entre las variedades estudiadas $(p>0,01)$. De acuerdo con la escala de severidad utilizada y a las condiciones en que se realizó este ensayo, todas las variedades presentaron valores promedio por debajo del grado 1, ubicándose en el mismo nivel de resistencia a la falsa mancha angular.

Por el contrario, sí fueron determinadas diferencias estadísticas en el desarrollo de la enfermedad de acuerdo con el tipo de hoja y edad de inoculación (Figura 2). El desarrollo de la enfermedad en hojas primarias fue más acelerada. El inicio de los síntomas ocurrió una semana posterior a la inoculación y la tasa de infección alcanzó su punto máximo en menos tiempo que las hojas trifoliadas. Esto es consecuencia de la mayor madurez de este tejido, que como se apuntó anteriormente, favorece la infección y reproducción del nematodo. El avance de la enfermedad fue constante y estuvo ligado a la disponibilidad de tejido, al grado de madurez del follaje, así como a la producción de inóculo secundario en las hojas primarias, en primera instancia, y luego en las hojas trifoliadas. 

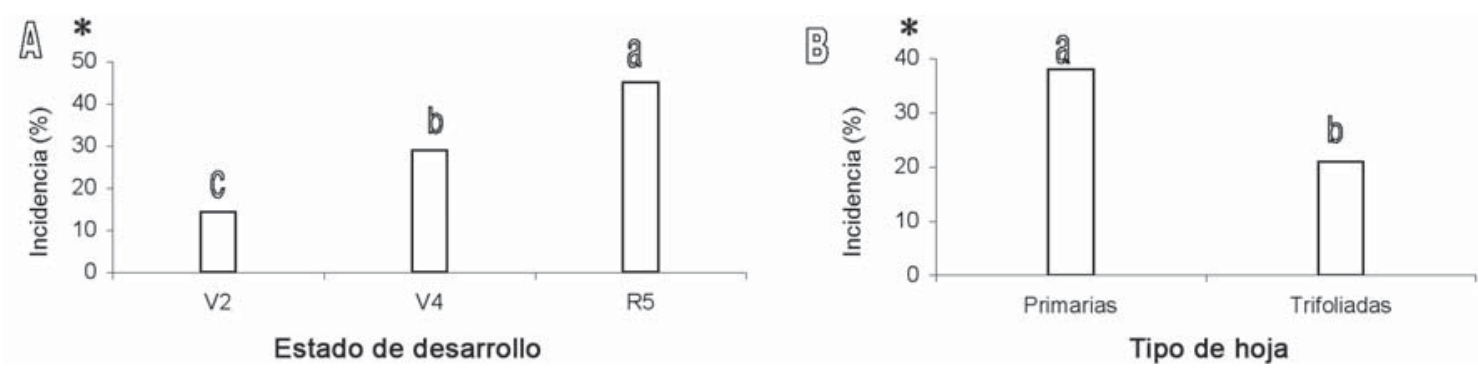

Figura 1. Incidencia de la FMA en frijol, en condiciones de invernadero. A) Efecto A. besseyi sobre diferentes estados fenológicos del cultivo, B) valores de incidencia según el tipo de hoja evaluada. Heredia, Costa Rica. 2006.

* Barras con diferente letra difieren estadísticamente, según prueba de $\operatorname{LSD}(\mathrm{p} \leq 0,05)$.
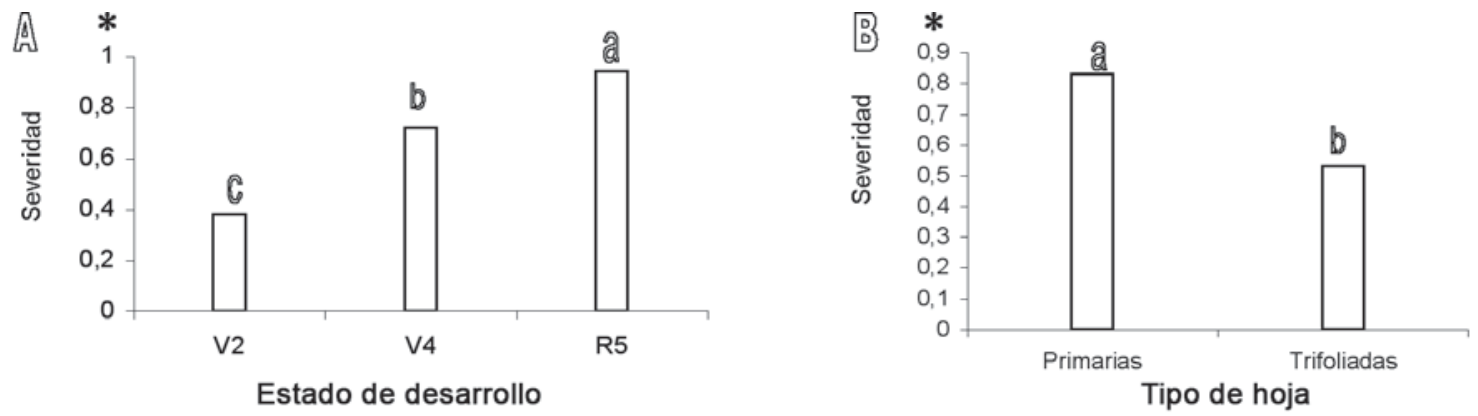

Figura 2. Severidad de la FMA, en condiciones de invernadero. A) Efecto A. besseyi sobre diferentes estados fenológicos del cultivo, B) valores de severidad según el tipo de hoja evaluado. Heredia, Costa Rica. 2006.

* Barras con diferente letra difieren estadísticamente, según prueba de LSD $(\mathrm{p} \leq 0,05)$.

Tanto la incidencia como la severidad y la población de nematodos, siempre fueron mayores a medida que aumentó la edad de las plantas inoculadas. Sobre este aspecto, Hesting y Wallace (1960), Christie (1974), Prado y Sosa-Moss (1978) y Lin et al. (1992), mencionan que Aphelenchoides spp. prefiere follaje maduro para iniciar su parasitismo; este tejido, generalmente ubicado en el dosel inferior de las plantas, aparentemente es más susceptible al ataque del nematodo. Según Salas y Vargas (1984), el mayor ataque del nematodo a hojas bajeras del frijol está relacionado con un porcentaje mayor de humedad en este estrato. Sin embargo, esto no es aplicable bajo condiciones de invernadero, donde el ambiente permaneció saturado de humedad durante el período del ensayo. Igualmente, podríamos pensar que bajo condiciones de campo la presencia de la enfermedad en hojas bajeras estaría relacionada con la cercanía a la fuente de inóculo y la diseminación por salpique de lluvia. De nuevo, esta situación no es aplicable bajo condiciones de invernadero, donde la inoculación se realizó directamente sobre el follaje y el riego fue por medio de nebulización. De lo expuesto anteriormente se podría inferir que la enfermedad aparece primero en las hojas primarias como consecuencia de factores relacionados con aspectos morfológicos, fisiológicos y arquitectónicos propios de este tipo de hojas, que las hacen más susceptibles con respecto a las hojas trifoliadas. 


\section{Población de nematodos extraídos del follaje}

Con respecto a la variable población de nematodos, el análisis estadístico mostró diferencias altamente significativas $(\mathrm{P}<0,001)$ entre variedades. La mayor cantidad de nematodos se extrajo de las variedades Telire y Chirripó Rojo. Además, la población de A. besseyi en las plantas aumentó conforme la edad del tejido inoculado, (estado fenológico R5, seguido por el estado V4 y finalmente el estado V2 como se observa en la Figura 3. Tsay et al. (1998) mencionan que la mayor tasa de nematodos de $A$. besseyi en arroz se obtiene cuando la planta alcanza su etapa reproductiva. Esta característica del nematodo en el cultivo de arroz concuerda con los resultados encontrados en este ensayo. La etapa de desarrollo del frijol R5 marca el inicio de la etapa reproductiva, período en el cual la planta destina la mayor parte de su energía en la producción de flores y frutos. Por consiguiente, los patógenos para encuentran pocas defensas en las plantas y se facilita la colonización de los tejidos. Las etapas donde el tejido de frijol está aún inmaduro (etapas vegetativas), aparentemente no ofrece ventajas al nematodo para su establecimiento.

Las variedades Huetar y Bribri presentaron las menores poblaciones de Aphelenchoides besseyi del ensayo, difiriendo estadísticamente de las variedades Telire y Chirripó Rojo, lo que les confirió un mayor grado de resistencia al parasitismo del nematodo bajo las condiciones en que se realizó este ensayo.

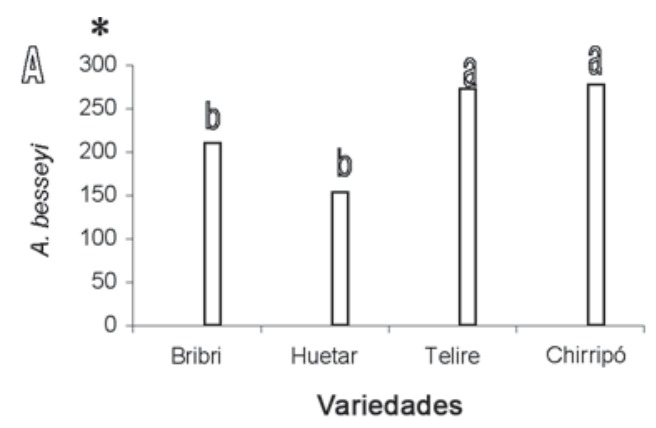

\section{CONCLUSIONES}

La duración del periodo de incubación de la falsa mancha angular del frijol en condiciones de invernadero, estuvo determinada por el tipo de hoja evaluada. Así, para hojas primarias, el ámbito obtenido fue de ocho a 14 días, mientras que de ocho a 24 días en hojas trifoliadas. Además, el periodo de incubación disminuyó en hojas trifoliadas conforme aumentó la edad de las plantas.

De acuerdo con la escala de severidad utilizada y a las condiciones en que se realizó este ensayo, las variedades comerciales de frijol Bribri, Huetar, Telire y Chirripó Rojo, se ubicaron dentro del mismo nivel de resistencia a la falsa mancha angular.

Las variedades Huetar y Bribri presentaron las menores poblaciones de Aphelenchoides besseyi del ensayo, difiriendo estadísticamente de las variedades Telire y Chirripó Rojo, lo que les confirió un mayor grado de resistencia al parasitismo del nematodo bajo las condiciones en que se realizó este ensayo.

La edad de inoculación de las plantas (V2, V4, R5), presentó diferencias estadísticas para las variables período de incubación, incidencia, severidad y población de nematodos; a medida que se aumentó la edad de las plantas éstas fueron más susceptibles al nematodo.

Figura 3. Número de A. besseyi, en condiciones de invernadero. A) Población de A. besseyi en las diferentes variedades estudiados, B) Efecto de los estados fenológicos sobre la población de nematodos. Heredia, Costa Rica. 2006.

\footnotetext{
* Barras con diferente letra difieren estadísticamente, según prueba de LSD ( $\mathrm{p} \leq 0,05)$.
} 
El desarrollo de la enfermedad describió un comportamiento diferente dependiendo del tejido estudiado: en el tejido primario el índice de severidad describe un ascenso pronunciado en las primeras semanas declinó rápidamente como consecuencia de la senescencia de este tejido. En el tejido trifoliado el índice de severidad inició más lentamente pero aceleró conforme maduraron, debido a la mayor disponibilidad de tejido maduro susceptible y más producción de inóculo secundario.

El ataque del nematodo al inició fue más severo en las hojas primarias, debido a que A. besseyi prefiere tejido maduro para iniciar su parasitismo. Además, la posición horizontal de las hojas primarias y su arquitectura favoreció la acumulación de humedad, elemento necesario en los procesos de reproducción y diseminación del nematodo.

Existió una alta correlación positiva entre las variables de incidencia, severidad y población de nematodos; por lo que cualquiera de las tres variables puede ser utilizada para medir el desarrollo de la enfermedad, facilitando el estudio de la enfermedad en futuras investigaciones.

\section{LITERATURA CITADA}

Agrios, GN. 1985. Fitopatología. México, DF, Limusa. p. 661-710.

Arauz, LF. 1998. Fitopatología: un enfoque agroecológico. San José, CR. Editorial Universidad de Costa Rica. p. 224-263.

Araya, CM; Hernández, JC. 2003. Distribución agroecológica de enfermedades del frijol en Costa Rica. Manejo Integrado de Plagas y Agroecología 68: 26-33.

Araya, R; Hernández, JC. 2007. Variedades de frijol de grano rojo obtenidas por Fitomejoramiento Participativo en Costa Rica 2000 - 2007. Fondo de Desarrollo de Noruega (FDN). San José, Costa Rica. 18 p.

Barrantes, W; Araya, CM. 2004. Falsa mancha angular del frijol; una enfermedad que avanza. In: Programa de Investigación y Transferencia de Tecnología
Agropecuaria - Frijol (PITTA-FRIJOl) “VIII Reunión anual del Sector Frijolero de Costa Rica. Costa Rica. ITCR-Santa Clara, San Carlos. p. 53-54.

Barrantes, W; Araya, CM; Esquivel, A. 2005. Incremento in vitro de Aphelenchoides besseyi. In: Memorias I Congreso Nacional de Fitoprotección. San José, CR. p. 66.

CIAT (Centro Internacional de Agricultura Tropical). Programa de frijol. 1982a. Principales nematodos que atacan el frijol y su control. Guía de estudio para ser usada como complemento de la Unidad Audiotutorial sobre el mismo tema. Contenido científico: Varón de A., Francia; Riedel M., Richard y Programa de Frijol del CIAT-Fitopatología. Producción: Ospina, Héctor F. y Flor, Carlos A. (Serie 04SB-06.10). Palmira, Colombia. 37 p.

CIAT (Centro Internacional de Agricultura Tropical). Programa de frijol. 1982b. Etapas de desarrollo de la planta de frijol común. Guía de estudio para ser usada como complemento de la Unidad Audiotutorial sobre el mismo tema. Contenido científico: Fernández, Fernando; Gepts, Paúl; López, Marceliano. Producción: Ospina O, Héctor F. Colaboración: Hidalgo, Rigoberto. (Serie 04SB-09.03). Palmira, Colombia. 26 p.

Christie, JR. 1974. Nematodos de los vegetales: su ecología y control. México, D.F., Editorial Limusa. 263 p.

Franc, GD; Beaupere, CM; Gray, FA; Hall, RD. 1996. Nematode angular leaf spot of dry bean in Wyoming. Plant Disease 80: 476-477.

Hesting, JJ; Wallace, HR. 1960. Susceptibility of varieties of chrysanthemum to infestation by Aphelenchoides ritzemabosi. Nematologica 5: 297-302.

Hoshino S; Togashi K. 2000. Effect of water-soaking and airdrying on survival of Aphelenchoides besseyi in Oryza sativa seeds. Journal of Nematology 32: 303-308.

Klingler, J. 1970. The relation of Aphelenchoides fragariae to slit-like micro-openings and to stomal diffusion gases. Nematologica 16: 417-422.

Lin, YY; Wang, KM; Tsay, TT. 1992. The occurrence of Aphelenchoides besseyi Christie on Dendrobium Lady Fay. Plant Protection Bulletin 34: 202-215. 
Prado, C; Sosa-Moss, C. 1978. Presencia en México de Aphelenchoides ritzemabosi en follaje de margariton. Nematropica 8: 6.

Salas,LA; Vargas, E. 1984.Elnematodo foliar Aphelenchoides besseyi Cristie (NEMATODE: aphelenchoididae) como causante de la falsa mancha angular del frijol en Costa Rica. Agronomía Costarricense 8: 65-68.

Tsay, TT; Cheng, YH; Teng, YC; Lee, MD; Wu, WS; Lin, YY. 1998. Bionomics and control of rice white tip disease nematode, Aphelenchoides besseyi. Plant Protection Bulletin 40: 277-286.

Todd, EH; Atkins, JG. 1958. White tip disease of rice. I. Symptoms, laboratory culture of nematodes, and pathogenicity tests. Phytopathology 48: 632-637.

Wallace, HR. 1960. Observations on the behaviors of Aphelenchoides ritzemabosi in chrysanthemum leaves. Nematologica 5: 315-321. 